\title{
LA VALIDEZ DE TRATAMIENTO DEL CUESTIONARIO BÁSICO DE DEPRESIÓN. PRIMEROS DATOS
}

\author{
Wenceslao Peñate* \\ Carmen T. P|tt|** \\ Auxllladora García* \\ Lllisbeth Perestelo"t* \\ Universidad de La Laguna* \\ Hospital Universitario de Canarias** \\ Servicio Canario de Saludeme
}

\section{RESUMEN}

Como parte del procedimiento de validación de una nueva medida de depresión, el Cuestionario Básico de Depresión (CBD), se presentan datos sobre su validez de tratamiento. El trabajo se inserta dentro del concepto psicométrico más general de validez incremental, analizando en qué medida el CBD aporta elementos especificos a la evaluación de la depresión. Con un diseño transversal, se evalúo a 101 pacientes con distintos tipos de depresión (depresión mayor, trastornos adaptativos, distimia...) con el CBD y el Inventario de Depresión de Beck-ll (BDI-II). El progreso terapéutico se evalúo por medio del juicio clínico de los terapeutas y por la opinión de los propios pacientes sobre su nivel de ajuste. Los resultados mostraron como el CBD permite monitorizar el progreso terapéutico de una manera similar a como lo hace el BDl-Il. La aportación del CBD a la validez de tratamiento indica que es un cuestionario que monitoriza mejor

Correspondencia: Wenceslao Peñate, Dto. de Personalidad, Evaluación y Tratamientos Psicológicos, Universidad de La Laguna, 38204 La Laguna (Teneríle). Correo electrónico: wpenate ull.es. 
los cambios terapéuticos cuando se toman en cuenta criterios clínicos de mejora y a la opinión de los propios pacientes. Los resultados se discuten de acuerdo a la sintomatología evaluada por el CBD y su capacidad para detectar los cambios más genuinos del sufrimiento depresivo.

Palabras clave: CUESTIONARIO BÁSICO DE DEPRESIÓN, VALIDEZ DE TRATAMIENTO, VALIDEZ INCREMENTAL, MUESTRA DE DEPRESIVOS.

\section{SUMMARY}

As part of the validity procedure of a new measure for depression, the Basic Questionnaire for Depression (CBD), we provide data about its treatment validity. This study is inserted in a broader psychometric concept, incremental validity, to analyse as the $C B D$ provides significant data about the assessment of depression. With a transversal design, 101 patients with different types of depression disorders (major depression, adaptative disorders, dysthymia...) were assessed with $C B D$ and Beck Depression Inventory (BDI-II). Therapeutic progress was assessed according to clinician judgment, and patient opinions about their level of adjustment. Results pointed out that CBD allows the control of therapeutic progress as BDI-II does it. CBD provides specific data about treatment validity when both clinical improvement criteria and the self-adjustment declared by patients are taking into account. Results are discussed according to the depressive symptoms assessed by CBD and its ability to detect crucial changes in depressive suffering

Key words: BASIC QUESTIONNAIRE FOR DEPRESSION, TREATMENT VALIDITY, INCREMENTAL VALIDTTY, DEPRESSIVE SAMPLE.

El Cuestionario Básico de Depresión (CBD) es un inventario construido con la finalidad de evaluar los síntomas genuinos de los trastornos depresivos (Peñate, 2001a). La sintomatología de estos trastornos es muy variada. A grandes rasgos, se podría identificar la sintomatología depresiva bajo tres atributos: (i) El síndrome depresivo está formado por diversos síntomas que también se encuentran en 
otros trastornos (actitudes negativas, trastornos neurovegetativos deterioro cognitivo...), lo que dificulta su diagnóstico diferencial, especialmente con algunos trastornos de ansiedad. (ii) Los sintomas tradicionalmente asociados al fenómeno depresivo (afecto triste, anhedonia) no constituyen síntomas obligatorios para su diagnóstico (APA, 2002; OMS, 1992). (iii) Paradójicamente, algunos síntomas son bidireccionales, constituyendo su valor para la depresión, no el síntoma en sí mismo, sino un atributo: su baja o elevada intensidad o frecuencia de ocurrencia (sueño, apetito, comportamiento psicomotor).

Desde hace tiempo existe un volumen importante de escalas e inventarios para evaluar la depresión. Sin embargo, la situación de la sintomatología y, consecuentemente, los problemas de validez de constructo, validez de contenido y de validez diferencial no parece haberse resuelto. Así, tal y como constataron hace ya bastante tiempo Levitt y Lubin (1975), cada escala e inventario obedece a unos criterios de validez de contenido especifica de cada modelo de depresión en el que se sustenta cada escala. Haciendo un análisis breve, en general las distintas escalas muestran unos buenos indicadores psicométricos en relación con su consistencia interna, su capacidad para diferenciar a depresivos frente a población no clínica, su validez convergente y su validez de tratamiento. Las dificultades han estado en su capacidad para el diagnóstico diferencial (especialmente con algunos trastornos de ansiedad) y su validez para discriminar entre distintos trastomos depresivos. Desde el punto de vista conceptual, se ha cuestionado la validez de constructo ante la dificultad de aislar un único componente depresivo.

Como ilustración de este estado de cosas se pueden citar a dos inventarios bien conocidos: la escala de depresión del MMPI (MMPI-D, Hathaway y McKinley, 1943) el Inventario de Depresión de Beck (BDI, Beck, Rush, Shaw y Emery, 1979; Beck, Ward, Mendelson, Mock y Erbaugh, 1961). Ambos inventarios han tenido y tienen un amplio uso, tanto clínico como de investigación, y ambos han sido revisados $y$ reelaborados hace poco tiempo (MMPI-2-D, Butcher, Dahlstrom, Graham, Tellegen y Kaemmer, 1989; y BDI-II, Beck, Steer y Brown, 1996).

Con respecto a la escala de de depresión del MMPI, ésta ha sido objeto de controversias fundamentadas en su sensibilidad (verdaderos positivos) y especificidad (verdaderos negativos), en la irrelevancia de 
muchos de sus contenidos e, incluso, en problemas de segregación racial (p.e., Greene, 1987). Las modificaciones del MMPI-2 han consistido en la eliminación de contenidos de la escala original (sólo tres ítems), y el añadido de una nueva escala que incluyera contenidos no abordados en primera versión. Estas nuevas escalas, sin embargo, no han terminado con esos problemas. Más aún, en un trabajo de meta-análisis llevado a cabo por Gros, Keyes y Greene (2000), donde revisaron 18 estudios que aplicaban los dos formatos del MMPI y seleccionados por cumplir una serie de requisitos metodológicos, los resultados no fueron mejores para la nueva versión: las tasas de sensibilidad y especificidad fueron similares para la vieja y las nuevas escalas. En todo caso las tasas mejores fueron para la escala original, lo que llevó a que la tasa de clasificaciones correctas también fuera superior para la versión de 1943.

Con respecto al BDI, se puede decir que es el inventario con mayor apoyo psicométrico hoy día. En 1996 se publicó una segunda versión, con la finalidad de adaptar el inventario a los síntomas de la depresión recogidos en la cuarta versión del DSM (APA, 1995). Tanto la versión original como la segunda versión han recibido apoyo empírico con respecto a su bondad psicométrica (Beck y cols., 1996; Beck, Steer y Garbin). Sin embargo, algunos problemas continúan, especialmente en lo referido en su capacidad para el diagnóstico diferencial frente a los trastornos de ansiedad y para discriminar entre distintos tipos de depresión (Steer, Brown, Beck, y Sanderson, 2001; Steer, Clark, Beck, y Ranieri, 1999; Storch, Roberti, y Roth, 2004). Por lo demás, su validez de constructo continúa apoyándose en un modelo jerárquico, con dos o tres elementos principales (p.e., Storch y cols., 2004), destacando las actitudes negativas o cognitivo-afectivas, y un factor general de depresión de segundo orden.

En este estado de las cosas es el que se realiza la propuesta de un nuevo cuestionario para evaluar la depresión: el CBD ha tratado de encontrar los síntomas genuinos de la depresión, que responda a los problemas conceptuales, al diagnóstico diferencial y a la validez de constructo. Para su construcción se apoyó en el modelo de parámetros (Pelechano, 1996, 2000). Así, se elaboró una prueba en la que inicialmente no se presupuso la existencia de síntomas primarios o secundarios, sino que se entendió que la sintomatologia covariaba 
para dar cuenta del fenómeno depresivo, no pudiéndose establecer para todos los procesos depresivos síntomas causativos y síntomas consecuenciales (principio de determinación recíproca); se construyó una prueba para evaluar los aspectos más estables de la depresión, evitando elementos de inestabilidad situacional (nivel de consolidación básico); y se elaboró un sistema de respuesta acorde a la naturaleza de lo evaluado, acorde con el curso temporal de la depresión (presencia de la sintomatología en semanas, meses o años).

En trabajos anteriores hemos tratado de aportar información sobre la bondad psicométrica de este nuevo inventario, mostrando una validez de constructo, fundamentada en tres contenidos de la depresión: el afecto triste, la anhedonia y la baja autoestima (Peñate, 2001b y c) ${ }^{1}$. Esta escala ha mostrado su utilidad en algunos aspectos criticos como han sido la validez diferencial entre ansiedad y depresión (Peñate, Ibáñez y González, 2001; González, Herrero, Viña, Ibáñez, y Peñate, 2004), la validez discriminante entre distintos tipos de trastornos depresivos (Peñate y Almeida, 2001), o su vinculación con los modelos explicativos más relevantes de la depresión (Peñate, Perestelo y Bethencourt, 2004).

Ahora, en ese sentido de aportar información psicométrica, se presentan datos sobre su validez de tratamiento. En este caso, se trata de conocer en qué medida el CBD es capaz de detectar los cambios que se producen en la situación de pacientes depresivos como consecuencia de algún típo de intervención: en qué medida permite monitorizar el proceso de intervención sobre la sintomatología depresiva. Pretendemos con ello insertarlo dentro de un concepto psicométrico más amplio, como es el de validez incremental.

Hace ya cierto tiempo (Cronbach y Gleser, 1957), se postulaba la necesidad de que el procedimiento de validación de una prueba no fuera algo estático, sino que fuera un proceso continuo que iría aportando datos que ajustaran y mejoraran la medida en cuestión. Posteriormente Sechrest (1963), iba a perfilar el concepto de validez incremental, añadiendo un elemento de comparabilidad. En este sentido, este tipo de validez hacia referencia a en qué medida una prueba mejoraba la capacidad predictiva en comparación con otra fuente de datos, sobre

' A propósito de estos contenidos, otra prueba reclente elaborada para evaluar depresión, el ST/DEP (Spielberger, Carretero-Dios, de los Santos, y Buela-Casal, 2002a y b), coincide en recoger elementos similares. 
un mismo objetivo. El interés no era tanto el de añadir datos de validación en general, sino el de incrementar la capacidad predictiva. Así, la validez incremental de un cuestionario que evaluara una dimensión determinada estribaba a en qué medida ese cuestionario permitiría hacer predicciones más ajustadas frente a los datos aportados por otros procedimientos (entrevistas, observación, datos de archivo...).

Aunque la validez incremental se ha aplicado a medidas en distintos ámbitos (diagnóstico psicológico, selección de personal, planificación del tratamiento, eficacia de los tratamientos...), tradicionalmente ha estado más ligado a la selección y asignación de puestos. Sin embargo, su importancia en los ámbitos de la medición psicológica es evidente, por al menos dos razones: (i) la necesidad de que la validación de una prueba sea un proceso dinámico, identificando progresivamente los mejores ámbitos de aplicación; y (ii) la existencia de numerosas medidas sobre un mismo fenómeno psicológico, haciendo necesaria una aclaración de qué es lo que aporta diferencialmente cada una y cuál es su poder predictivo frente a las otras pruebas existentes.

Este interés se vió reflejado en un número monográfico de la revista de la sección de evaluación psicológica de la APA (Psychological Assessment) en el año 2003. En este número se trató de ajustar y actualizar el concepto psicométrico y su aplicación a diferentes ámbitos. Para nuestro interés, Haynes y Lench (2003), consideran que la validez incremental de una prueba incluye tres elementos: lograr una capacidad predictiva más precisa, que esa contribución a la predicción sea significativa y que la prueba suponga unos menores costes, no sólo financieros, sino personales o facilitadores del proceso de evaluación. Específicamente, Hunsley y Meyer (2003) identifican tres tipos de validez incremental: la que hace referencia a la capacidad evaluativa y que tiene que ver con el concepto hasta aquí manejado referido a la mejora en la predicción de un criterio clínico relevante; la capacidad inferencial, referida a en qué medida los datos aportados por una prueba permiten al evaluador hacer juicios diagnósticos y tomas de decisiones más precisas; y la validez de nuevas medidas, para valorar en qué medida la propuesta de una nueva prueba sobre algún fenómeno psicológico aporta información significativa sobre las pruebas existentes (añade contenidos más adecuados, delimita mejor el concepto 
evaluado, añade aspectos no disponibles hasta ese entonces...).

Nuestra propuesta de un nuevo cuestionario para evaluar el fenómeno depresivo se ha situado inicialmente en este último tipo de validez incremental, en la medida en que nuestro cuestionario ha tratado de añadir algún elemento significativo en la evaluación de la depresión. Hemos tratado de identificar los contenidos más precisos de la depresión y hemos ido añadiendo datos de validación que mejoraran la capacidad predicativa de la nueva medida.

Ahora, y en ese mismo sentido de la validez incremental comentada, el objetivo general de este trabajo consiste en contrastar la validez de tratamiento del CBD: conocer si el CBD es capaz de detectar los cambios terapéuticos que se van produciendo en una muestra de depresivos que reciben tratamiento por su trastorno, y si esa detección es comparable a la que hace una prueba psicométricamente tan robusta como es el BDI-II.

Nuestras predicciones nos indican que es esperable que el CBD sea menos sensible a los cambios en la sintomatología depresiva. La razón estriba en que el CBD sólo evalúa tres síntomas depresivos (aquellos que conforman el afecto triste, la anhedonia y la baja autoestima); mientras que el BDI-II rastrea gran parte de los contenidos de la depresión (de acuerdo con los criterios del DSM-IV). Por lo tanto, es más probable que el BDI-II detecte cualquier cambio en los síntomas, mientras que el CBD sólo detectará esos cambios si se producen en las tres áreas señaladas. Sin embargo, secundariamente pensamos que los cambios detectados por el CBD tendrían un mayor alcance en la medlda en que son cambios que afectan a lo más genuino de la depresión.

\section{MÉTODO}

\section{Participantes}

Se evaluaron a 101 personas con algún diagnóstico de trastorno depresivo, procedentes de la Unidad de Salud Mental de Santa Cruz de Tenerife ${ }^{2}$. 95 de ellas eran mujeres y 6 hombres, con un rango de

\footnotetext{
2 Nuestro agradecimiento al personal de psiquiatría, psicología y enfermería de la USM del antiguo hospital psiquiátrico que amablemente colaboró en el pase de las pruebas y en la cumplimentación de datos adicionales.
} 
edad entre los 17 y los 61 años, siendo la media de 39 años. En cuanto al diagnóstico de los participantes, la distribución fue la siguiente (según el DSM-IV TR, APA, 2002): 47 fueron diagnosticados con un trastorno o episodio depresivo mayor (F32 y F33, con niveles moderados y graves). 27 padecían un trastorno adaptativo (con síntomas depresivos, F43.20, y mixto ansioso-depresivo, F43.22). 17 fueron diagnosticados con un trastorno distimico (F34.1). El resto de participantes (10), fueron diagnosticados con otros trastornos de carácter depresivo. En cuanto a la prevalencia del trastorno, 72 participantes informaron de una duración de menos de un año, mientras que 29 informaron de una duración de un año o más. Al analizar los antecedentes familiares de los participantes, 65 informaron que no tenían antecedentes del trastorno en su familia, mientras que 36 tenían un familiar de primer grado con un diagnóstico similar.

La mayoría de ellos recibían tratamiento farmacológico: 60 participantes estaban recibiendo tratamiento con algún inhibidor selectivo de los recaptadores de la serotonina (ISRS), 19 estaban siendo medicados con otros fármacos (tricíclicos e I-Mao, básicamente). Sólo 22 no recibian tratamiento farmacológico. Con respecto a tratamiento psicoterapéutico, la mayoria de la muestra (77 pacientes) recibía algún tipo de terapia de apoyo no especificada; 5 pacientes recibían algún tipo de terapia cognitivo-conductual (terapia de reestructuración cognitiva, terapia racional-emotiva...); y 19 pacientes no recibian ningún tipo de psicoterapia.

\section{Instrumentos}

Tal y como se menciona en la introducción, se administraron dos inventarios para evaluar el nivel de depresión: el Cuestionario Básico de Depresión y la segunda versión del Inventario de Depresión de Beck - Asimismo, para valorar la evolución de la sintomatología en los pacientes, se ha contado con información procedente del terapeuta y con información ofrecida por los propios pacientes. El terapeuta cumplimentaba lo que denominamos Protocolo de Control. Los pacientes cumplimentaban la Escala de Adaptación (Echeburúa y Corral, 1987, recogido en Borda y Echeburúa, 1991). 
El Cuestionario Básico de Depresión (Peñate, 2001a) es un cuestionario de 21 items con cuatro alternativas de respuesta sobre la duración de los síntomas que se mencionan (ninguna, semanas, meses, años). Este formato de respuesta se ha modificado con el fin de apresar los posibles cambios que se producen en la sintomatología en periodos más cortos de tiempo. Dado que se pretende detectar cambios, el formato se ha ajustado a cómo se siente y piensa la persona evaluada en las dos últimas semanas : no presencia de los síntomas, poca, bastante y mucha. Del mencionado trabajo (Peñate, 2001a) se puede observar que la escala presenta unos niveles de consistencia interna para muestras normales y de depresivos por encima de 0,80 , una estabilidad temporal elevada, conformando sus 21 items una estructura monofactorial.

Desde un punto de vista de clasificación psicopatológica se han establecido provisionalmente los siguientes criterios ${ }^{4}$ : de 0 a 19 , ausencia de depresión; de 20 a 29, depresión leve; de 30 a 39, depresión moderada; $y$ de 40 en adelante, depresión grave.

Inventario de Depresión de Beck, segunda versión (BDI-II, Beck, Steer y Brown, 1996; versión castellana de Sanz, Navarro y Vázquez, 2003; Sanz, Perdigón, y Vázquez, 2003). Este es un inventario basado en el inventario original del primer autor (Beck y cols., 1961; Beck y cols., 1979), se fundamenta en el modelo teórico de Aaron Beck para la depresión y está caracterizado por un alto contenido en distorsiones cognitivas (pesimismo, desesperanza, culpabilidad...). La segunda versión se ha llevado a cabo con la finalidad de adaptarlo a los criterios para el diagnóstico de los trastornos depresivos del DSM-IV (1995). Consta de 21 items con cuatro alternativas de respuesta (dos ítems tienen 7 alternativas). Por los datos aportados por sus autores, las propiedades psicométricas tanto de la primera (p.e., Beck y cols., 1988), como de la segunda versión (p.e., Beck y cols. 1996; Steer y cols., 2001), muestran unos adecuados indices de fiabilidad y validez.

3 Se ha tomado el criterio de dos semanas porque es el criterio temporal mínimo que reconocen las clasificaciones psicopatológicas para validar la presencia de síntomas depresivos.

4 Para obtener una puntuación global se ha establecido una puntuación de 0 a 3 . dependiendo de la alternativa de respuesta elegida. 
Con respecto a los criterios clínicos, los puntos de corte considerados son los siguientes: de 0 a 13, ausencia de depresión (depresión mínima); de 14 a 19, depresión leve; de 20 a 28, depresión moderada; de 29 en adelante, depresión grave.

Protocolo de control: Este protocolo era cumplimentado por los clínicos que tomaban a cargo al paciente. Su finalidad principal era la de conocer la evolución de la sintomatología depresiva, según el terapeuta. Secundariamente, se recababa información diversa sobre aspectos relevantes de cada paciente. En el anexo es recoge un ejemplar de este protocolo.

En primer lugar, se recababa información socio-demográfica y sanitaria. Datos de identificación (edad, género, ocupación, situación laboral y diagnóstico); información relacionada con el diagnóstico de los pacientes, con las siguientes categorías: trastorno o episodio depresivo mayor; trastorno distímico; trastorno adaptativo; trastorno bipolar; trastorno ansioso-depresivo y una última categoría que recoge otro tipo de trastornos no especificados. A continuación, el terapeuta cumplimentaba la información referente a la gravedad (en una escala de 5 puntos, donde $1=$ leve y $5=$ severa); la prevalencia (menos de un año, un año o más, dos años o más, tres años o más); la comorbilidad del trastorno; la existencia o no de antecedentes familiares (de primer y/o segundo grado); los tratamientos que el paciente ha recibido o recibe, y si alguna vez ha estado hospitalizado debido al trastorno.

En segundo lugar, el terapeuta cumplimentaba los datos sobre la situación actual: tratamiento psicológico (si recibe o no psicoterapia y, en caso afirmativo, qué tipo); tratamiento con psicofármacos; fase terapéutica en la que se encuentra el paciente: fase de evaluación, fase inicial (primer mes), fase de tratamiento y fase de seguimiento). Una vez especificada la fase se le requerla que emitiera un juicio sobre la evolución del paciente, en dos sentidos: la situación clínica actualy su nivel de adaptación. En la primera de ellas, el terapeuta evaluaba la evolución del paciente según su criterio (para las fases inicial, tratamiento y seguimiento). La escala estaba compuesta por cinco alternativas: sin cambios apreciables, algún cambio sin significación clínica, algún cambio clínicamente significativo, cambios clínicamente significativos, y en remisión total de síntomas. Con respecto al nivel de adaptación, se instruyó a los terapeutas para que, con la información 
ofrecida por el propio paciente, por sus familiares y otras personas externas a él, valoraran su evolución en cuatro parámetros: adaptación laboral/escolar, adaptación social, adaptación familiar, y ocio y tiempo libre. Con estos dos indicadores se valoraba la opinión de los facultativos sobre el progreso terapéutico en cada paciente.

Escala de Adaptación (Echeburúa y Corral, 1987). Es una escala que cumplimenta el propio paciente y que consta de 6 items que evalúan la adaptación en las siguientes áreas: funcionamiento en el trabajo y/o estudios; vida social habitual; actividades de ocio y tiempo libre; relación de pareja; relación familiar y funcionamiento global. Cada uno de los items es valorado de acuerdo con un formato de 6 alternativas, desde nada a muchisimo.

\section{Procedímiento}

Por medio de una de las psicólogas de la Unidad de Salud Mental de referencia, se contactó con el personal de psiquiatria, psicología y enfermería. En una reunión sobre este trabajo se les solicitó su participación voluntaria para que administraran una serie de pruebas a sus pacientes con algún tipo de depresión (con o sin comorbilidad): la escala de adaptación, el BDI-Il y el CBD. Al mismo tiempo se les explicaba la parte que cada uno debía cumplimentar para cada paciente (protocolo de control). Posteriormente se les dio instrucciones de cómo cumplimentar cada prueba.

Con respecto a cada paciente, se les instruyó para que para que informaran de que se trataba de un trabajo de investigación, garantizando la confidencialidad de la información y el uso grupal de la misma (nunca individualizado). Si el paciente accedía a participar, se le pedía su consentimiento informado.

Con respecto a los terapeutas, se les instruyó sobre el protocolo de control, acentuando tres cuestiones: fase en la que estaba el paciente, situación clínica y nivel de adaptación. Pro lo que se refiere a la fase, se consideró oportuno distinguir en la fase de tratamiento una fase inicial (primer mes, donde se pueden estar observando los primeros progresos terapéuticos), de la fase de tratamiento propiamente dicha. Las cuatro fases fueron: I fase, previa al tratamiento, cuando el paciente acude a la unidad y se le diagnóstica con algún trastorno depresivo; II fase, fase 
inicial del tratamiento. III fase, es la fase en la que el tratamiento puede estar siendo clínicamente efectivo. IV fase, seguimiento, una vez que el tratamiento ha sido efectivo y acude a sesiones de seguimiento. No obstante, se les instrula en el sentldo de que no era necesario que el tratamiento estuviera siendo efectivo para recoger los datos.

En ese sentido, se les solicitaba que indicaran la situación clínica, según su mejor criterio. Se les instruyó para que observaran cambios clínicamente significativos cuando consideraran que los síntomas detectados en cada paciente remitían (alguno, gran parte de ellos, todos). Con respecto a la escala de adaptación, se les pidió que valoraran el ajuste laboral/escolar, social, familiar, y ocio y tiempo libre. Para emitir este juicio tendrían en cuenta la tanto la información suministrada por cada paciente como por otras personas (familiares u otras personas significativas).

Durante tres meses se fueron recabando datos de los pacientes que voluntariamente quisieron colaborar. Una vez aceptada la colaboración, el terapeuta le entregaba las tres pruebas y se le daba las instrucciones de cumplimentación. El terapeuta por su parte cumplimentaba el protocolo de control y lo introducía, conjuntamente con las pruebas del paciente, en un sobre en el figuraba el código de identificación. Posteriormente se grabaron los datos y se llevaron a cabo los análisis estadísticos.

\section{RESULTADOS}

Un primer análisis se llevó a cabo para conocer las relaciones entre sí de las dos medidas de depresión y el grado de congruencia entre la opinión de los terapeutas y los pacientes con respecto a la evolución de su estado. Para ello se llevó a cabo un análisis correlacional (tipo Pearson). Con respecto a las relaciones entre el BDI-Il y el CBD el coeficiente se elevó a $0,80(p=0,001)$, mostrando una elevada validez convergente entre ambas medidas.

Por otro lado, el coeficiente de correlación entre el sexto ítem de la Escala de Adaptación (valoración global según el paciente) ${ }^{5}$ y el ítem

- Las puntuaciones de los items de la Escala de Adeptación se han invertido para una mejor comprensión de los resultados. De esta forma, a mayor puntuación mejor adaptación, según el propio paciente. 
'situación terapéutica' según el terapeuta fue de $0,55(p=0,01)$, indicando una significativa coincidencia entre ambas valoraciones. Por lo que se refiere a las valoraciones de aspectos parciales de la vida diaria, los coeficientes obtenidos se resumen en la tabla 1.

Tabla 1. Coeficientes de correlación (tipo Pearson) entre la valoración del paciente sobre su nivel de adaptación a distintas áreas de funcionamiento personal y la valoración del terapeuta $(n=101)$

\begin{tabular}{|c|c|c|c|c|c|}
\hline & $\begin{array}{l}\text { Nivel de ac } \\
\text { los pacion }\end{array}$ & taclón p & reas de $f$ & Ionamien & según \\
\hline $\begin{array}{l}\text { Adaptació } \\
\text { n según } \\
\text { terapeuta }\end{array}$ & laboral & social & ocio & familia & pareja \\
\hline laboral & $0,46\left(^{* *}\right)$ & $0,34\left({ }^{* *}\right)$ & $0,50\left(^{(*)}\right.$ & 0,23 & $0,33(* *)$ \\
\hline social & $0,50\left(^{* *}\right)$ & $0,46\left({ }^{(*)}\right)$ & $0,64\left(^{(*)}\right.$ & 0,26 & $0,44\left(^{(* *)}\right.$ \\
\hline ocio & $0,48(*+)$ & $0,46\left({ }^{(*)}\right)$ & $0,62\left(^{* *}\right)$ & 0,25 & $0,399^{(* *)}$ \\
\hline familia & $0,37\left(^{* *}\right)$ & $0,36\left({ }^{(*)}\right)$ & $0,50\left(^{(* *)}\right.$ & $0,36\left(^{* *}\right)$ & $0,32\left(^{(*)}\right.$ \\
\hline
\end{tabular}

* La correlación es significativa al nivel 0,01 (bilateral).

* Signiflcación al nivel de 0,05.

Como puede observarse, los niveles de congruencia entre los terapeutas y los pacientes son apreciables, aunque los mayores niveles de coincidencias se dan en los ámbitos de ocio y tiempo libre, y adaptación social, siendo la adaptación familiar la que menor acuerdo proporciona.

Con estos datos en mente, se procedió a los siguientes grupos de análisis: conocer los coeficientes de correlación entre la puntuación total en las dos medidas de depresión (BDI-Il y CBD) y las medidas de adaptación y progreso terapéutico. En la tabla 2 se resumen los coeficientes obtenidos entre las dos medidas de depresión y la opinión de los terapeutas sobre el progreso terapéutico.

Como era esperable, los coeficientes encontrados muestran en todos los casos una correlación negativa y significativa entre las puntuaciones entre distintas áreas y las puntuaciones en las dos medidas de depresión: cuanto mayor es el nivel de adaptación, menor es la puntuación en las escalas de depresión. Por inventarios, se observa una mayor potencia en el BDI-II en comparación con el CBD 
(sus coeficientes están alrededor de 0,50, llegando a 0,60 en el caso de la valoración global), aunque en todos los casos los niveles de significación son idénticos.

Con respecto a los coeficientes encontrados entre las medidas de depresión y la propia versión del paciente sobre sus niveles de adaptación, los resultados obtenidos se resumen en la tabla 3.

Tabla 2. Coeficientes de correlación (tipo Pearson) entre la valoración de los terapeutas sobre el progreso terapáutico en distintas áreas de

funcionamiento personal y las puntuaciones totales de dos medidas de depresión $(n=101)$

\begin{tabular}{|c|c|c|c|c|c|}
\hline & $\begin{array}{c}\text { adaptación } \\
\text { laboral }\end{array}$ & $\begin{array}{l}\text { adaptación } \\
\text { 8oclal }\end{array}$ & $\begin{array}{l}\text { adaptaclón } \\
\text { oclo }\end{array}$ & $\begin{array}{c}\text { adaptación } \\
\text { famillar }\end{array}$ & $\begin{array}{l}\text { Valoración } \\
\text { global }\end{array}$ \\
\hline$\overline{B D H}-11$ & $-0,43$ (**) $^{* *}$ & $-0,54\left(^{* *}\right)$ & $-0,499^{(*)}$ & $-0,46\left(^{* *}\right)$ & $-0,60\left({ }^{(*)}\right)$ \\
\hline CBD & $-0,38\left(^{\star \star}\right)$ & $-0,53\left(^{(*)}\right)$ & $-0,46\left({ }^{* *}\right)$ & $-0,38\left(^{* *}\right)$ & $-0,48\left(^{(*)}\right)$ \\
\hline
\end{tabular}

** La correlación es significativa al nivel 0,01 (bilateral); BDI-II = Puntuación en el Inventario de Depresión de Beck; CBD = Puntuación en el Cuestionario Básico de Depresión.

Tabla 3. Coeficientes de correlación (tipo Pearson) entre la valoración de los pacientes sobre el progreso terapáutico en distintas áreas de funcionamiento personal y las puntuaclones totales de dos medidas de depreslón $(n=101)$

\begin{tabular}{|l|c|c|c|c|c|c|}
\hline & $\begin{array}{c}\text { Adaptación } \\
\text { laboral }\end{array}$ & $\begin{array}{c}\text { Adaptaclón } \\
\text { social }\end{array}$ & $\begin{array}{c}\text { Adaptación } \\
\text { oclo }\end{array}$ & $\begin{array}{c}\text { Adaptación } \\
\text { tamiliar }\end{array}$ & $\begin{array}{c}\text { Adaptación } \\
\text { pareja }\end{array}$ & $\begin{array}{c}\text { Adaptación } \\
\text { global }\end{array}$ \\
\hline BDI-II & $-0,46\left(^{* *}\right)$ & $-0,56\left(^{* *}\right)$ & $-0,70\left(^{* *}\right)$ & $-0,36\left(^{* *}\right)$ & $-0,61\left(^{* *}\right)$ & $-0,64\left(^{* *}\right)$ \\
\hline CBD & $-0,40\left(^{* *}\right)$ & $-0,55\left(^{* *}\right)$ & $-0,70\left(^{* *}\right)$ & $-0,34\left(^{(*)}\right)$ & $-0,54\left({ }^{* *}\right)$ & $-0,52\left({ }^{* *}\right)$ \\
\hline
\end{tabular}

** La correlación es significativa al nivel 0,01 (bilateral); BDI-II = Puntuación en el Inventario de Depresión de Beck; CBD = Puntuación en el Cuestionano Básico de Depresión.

De nuevo, el patrón de correlaciones es similar al obtenido con la opinión de los terapeutas. En este caso, además, los coeficientes de correlación del BDI-II y el CBD son de cuantías similares, mostrando cómo ambas medidas tienden a disminuir significativamente cuando las personas valoran un mayor nivel de adaptación e, inversamente, los niveles de depresión aumentan a medida que disminuye el nivel de adaptación. Por lo demás, llama la atención que el coeficiente con 
mayor valor absoluto se obtiene entre las dos medidas de depresión y la adaptación al ocio y tiempo libre $(0,70)$, y la menor con la adaptación familiar.

Una vez observada la relación entre los criterios de adaptación/ mejora terapéutica y las puntuaciones en las dos medidas de depresión, se quiso conocer la capacidad de estas medidas para discriminar en distintos grupos clínicos de mejora, tanto para el criterio del terapeuta como para el propio criterio del paciente.

Con respecto al criterio del terapeuta, se estableció una medida compuesta por los cuatro criterios de mejora evaluados (adaptación laboral, social, ocio y familiar) y la valoración de la situación clínica, reconvirtiéndola en tres niveles: no mejora (coincidencia entre los niveles de adaptación y la valoración clínica de sin cambios apreciables); mejora ligera (coincidencia de los niveles de adaptación con los criterios clínicos de algún cambio sin significación clínica y algún cambio clínicamente significativo); y mejora apreciable, coincidencia entre los niveles de adaptación y las valoraciones cambios clínicamente significativosy en remisión total de sintomas). Este supuso que algunos pacientes no pudieran ser clasificados. Asi, se dispuso de 29 pacientes identificados con no mejora, 30 con mejora ligera y 20 con mejoras apreciables. En la tabla 4 se resume el análisis de varianza para estos tres grupos sobre las puntuaciones en el BDI-II y el CBD.

Tabla 4. Resumen del análisis de varianza de tres medidas de mejora según el terapeuta (no mejora, mejora ligera y mejora apreciable) sobre las puntuaciones en el Inventario de Depresión de Beck (BDI-II) yel Cuestionario Básico de Depresión (CBD). Para el contraste entre grupos se utilizó la T de Tukey

\begin{tabular}{|c|c|c|c|c|c|c|}
\hline & $\begin{array}{l}\text { Fuente de } \\
\text { varlaclón }\end{array}$ & $\begin{array}{l}\text { Suma de } \\
\text { cuadrados }\end{array}$ & $\begin{array}{c}\text { Grados de } \\
\text { llbertad }\end{array}$ & $\begin{array}{c}\text { Medla } \\
\text { cuadrátlce }\end{array}$ & $\mathbf{F}$ & Sig. \\
\hline BDI-II & Inter-grupos & 2992,60 & 2 & 1496,30 & \multirow[t]{3}{*}{28,74} & \multirow[t]{3}{*}{0,001} \\
\hline & Intra-grupos & 4165,40 & 80 & 52,07 & & \\
\hline & Total & 7158,00 & 82 & & & \\
\hline \multirow[t]{3}{*}{ CDB } & Inter-grupos & 3308,01 & 2 & 1654,00 & \multirow{3}{*}{19,22} & \multirow{3}{*}{0,001} \\
\hline & Intra-grupos & 6627,48 & 77 & 86,07 & & \\
\hline & Total & 9935,49 & 79 & & & \\
\hline
\end{tabular}


Tabla 4. Resumen del análisis de varianza de tres medidas de mejora según el terapeuta (no mejora, mejora ligera y mejora apreciable) sobre las

puntuaciones en el Inventario de Depresion de Beck (BDI-II) y el Cuestionario Básico de Depresión (CBD). Para el contraste entre grupos se utilizó la T de Tukey (continuación)

\begin{tabular}{|c|c|c|c|c|c|}
\hline & Grupos & $\mathbf{N}$ & Medla & $\begin{array}{c}\text { Desvlació } \\
\text { n típlca }\end{array}$ & $\begin{array}{l}\text { Contrastes } \\
\text { múltiples }\end{array}$ \\
\hline \multirow[t]{3}{*}{ BDI-II } & 1. No mejora & 29 & 36,93 & 5,61 & \multirow{3}{*}{$\begin{array}{l}1=2 \\
1<3 \\
2<3\end{array}$} \\
\hline & 2. Mejora ligera & 34 & 33,91 & 6,58 & \\
\hline & 3. Mejora apreciable & 20 & 21,60 & 9,88 & \\
\hline \multirow[t]{3}{*}{ CDB } & 1. No mejora & 29 & 38,39 & 8,82 & \multirow{3}{*}{$\begin{array}{l}1=2 \\
1<3 \\
2<3\end{array}$} \\
\hline & 2. Mejora ligera & 34 & 38,50 & 8,48 & \\
\hline & 3. Mejora apreciable & 20 & 23,60 & 10,99 & \\
\hline
\end{tabular}

Tabla 4. Resumen del análisis de varianza de tres medidas de mejora según el paciente (no mejora, nivel intermedio y mejora) sobre las puntuaciones en en el Inventanio de Depresión de Beck (BDI-II) y el Cuestionario Báslco de Depresión (CBD). Para el contraste entre grupos se utilizo la T de Tukey

\begin{tabular}{|c|c|c|c|c|c|c|}
\hline & $\begin{array}{l}\text { Fuente de } \\
\text { varjación }\end{array}$ & $\begin{array}{l}\text { Suma de } \\
\text { cuadrados }\end{array}$ & $\begin{array}{c}\text { Grados de } \\
\text { llbertad }\end{array}$ & $\begin{array}{l}\text { Medla } \\
\text { cuadrátlca }\end{array}$ & $\mathbf{F}$ & Sig. \\
\hline \multirow[t]{3}{*}{ BDHII } & Inter-gnupos & 3995,72 & 2 & 1997,86 & \multirow{3}{*}{40,51} & \multirow{3}{*}{0,001} \\
\hline & Intra-grupos & 3797,08 & 77 & 49,31 & & \\
\hline & Total & 7792,80 & 79 & & & \\
\hline \multirow[t]{3}{*}{ CDB } & Inter-grupos & 4568,17 & 2 & 2284,09 & \multirow{3}{*}{32,94} & \multirow{3}{*}{0,001} \\
\hline & Intra-grupos & 5061,99 & 73 & 69,34 & & \\
\hline & Total & 9630,16 & 75 & & & \\
\hline
\end{tabular}

\begin{tabular}{|c|c|c|c|c|c|}
\hline & Grupos & $\mathbf{N}$ & Media & $\begin{array}{c}\text { Desvlación } \\
\text { típlca }\end{array}$ & $\begin{array}{l}\text { Contrastes } \\
\text { múltiples }\end{array}$ \\
\hline \multirow[t]{3}{*}{ BDI-II } & 1. No mejora & 19 & 39,89 & 4,358 & \multirow{3}{*}{$\begin{array}{l}1<2 \\
1<3 \\
2<3\end{array}$} \\
\hline & 2. Intermedio & 41 & 34,29 & 6,86 & \\
\hline & 3. Mejora & 20 & 20,60 & 9,10 & \\
\hline \multirow{3}{*}{ CDB } & 1. No mejora & 18 & 41,78 & 6,98 & \multirow{3}{*}{$\begin{array}{l}1=2 \\
1<3 \\
2<3\end{array}$} \\
\hline & 2. Intermedio & 40 & 37,15 & 8,76 & \\
\hline & 3. Mejora & 18 & 20,89 & 8,55 & \\
\hline
\end{tabular}


Los contrastes muestran unas claras diferencias significativas entre los tres grupos de mejora y las puntuaciones tanto en el BDI-II y el CBD. Sin embargo, cuando se contrastan los tres grupos dos a dos ( $T$ de Tukey), no se observan diferencias entre el grupo de no mejora y el de mejora ligera, tanto para el BDI-II como para el CBD. Las diferencias se establecen este estos dos grupos y el grupo de mejora apreciable.

Con respecto a la opinión de los pacientes, se construyó un índice con el sumatorio de las cinco áreas de funcionamiento autoevaluadas (adaptación laboral, social, ocio, familia y pareja). Los pacientes agrupados hasta el percentil 25 se consideraron como 'no mejora', entre el percentil 25 y el 75 'nivel intermedio' y los pacientes situados en el percentil 75 o superior se consideraron el grupo de 'mejora' (tamblén en este caso se perdieron datos de algunos pacientes, debido a que el algunos casos dejaron de responder a algún ítem de la escala deadaptación). Estos tres grupos se contrastaron en las puntuaciones en el BDI-II y CBD. En la tabla 5 se resumen estos contrastes.

El patrón de resultados es similar al realizado con la opinión del terapeuta, mostrando que tanto el BDI-II como el CBD monitorizan significativamente los tres estadlos de cambio evaluados. En este caso, además, el BDI-II incluso logra discriminar entre los niveles de no mejora y de nivel intermedio de mejora. Sin embargo, el CBD continúa sin discriminar entre esas dos medidas.

Si observamos las puntuaciones medias de los grupos de mejora creados en estos dos últimos análisis, destaca un aspecto importante: las puntuaciones promedio en depresión de las medidas consideradas como de 'mejora apreciable' por parte del terapeuta o de 'mejora' por parte de los propios pacientes, continúan siendo clínicamente relevantes. Tomando como criterio el BDI-II (Beck y cols., 1996), las puntuaciones promedio del grupo de mejora terapéutica se sitúa alrededor de 20, lo que indicaría unos niveles de depresión moderado. Esto podía estar indicando que los criterios de mejora tomados por nosotros hacian más referencia a un criterio actuarial que clínico. En este sentido, se llevaron a cabo nuevos análisis, agrupando las medidas realizadas de acuerdo con los cuatro grupos clínicos que permite el $\mathrm{BDI}-\mathrm{Il}$ (no depresión, depresión leve, moderada y grave) y los cuatro grupos similares con el CBD. El criterio de mejora según los terapeutas 
se mantuvo idéntico (porque se fundamentaban en el juicio sobre la situación clínica), pero el nivel de mejora por parte de los propios pacientes se modificó en un sentido clínico, agrupando las puntuaciones en la escala de adaptación en tres niveles: no mejora (puntuaciones promedio 5 y 6 en la escala de adaptación), nivel intermedio (puntuaciones 3 y 4), y mejora (puntuaciones 1 y 2). Los contrastes realizados se llevaron a cabo por medio de tablas de contingencias. En la tabla 5 se resumen los datos entre el BDI-II y las dos medidas de mejoría terapéutica.

Tabla 5. Tablas de contingencia entre los cuatro grupos clínicos de acuerdo con la puntuación obtenida en el BDI-ll y las opiniones de progreso

terapético emitidos por los terapeutas y los proplos pacientes

\begin{tabular}{|l|r|r|r|}
\hline \multirow{2}{*}{ Grupos clínicos } & \multicolumn{3}{|c|}{ Progreso según los terapeutas } \\
\cline { 2 - 4 } & No mejora & \multicolumn{1}{|c|}{ Mejora ligera } & \multicolumn{1}{c|}{ apreciable } \\
\hline Sin depresión & $0,0 \%$ & $0,0 \%$ & $100,0 \%$ \\
\hline Leve & $0,0 \%$ & $33,3 \%$ & $66,7 \%$ \\
\hline Moderada & $12,5 \%$ & $31,3 \%$ & $56,3 \%$ \\
\hline Grave & $45,8 \%$ & $47,5 \%$ & $6,8 \%$ \\
\hline
\end{tabular}

\begin{tabular}{|l|r|r|r|}
\hline \multirow{2}{*}{ Grupos clínicos } & \multicolumn{3}{|c|}{ Nivel de adaptación según los paclentes } \\
\cline { 2 - 4 } & No mejora & Nivel intermedio & \multicolumn{1}{c|}{ mejora } \\
\hline Sin depresión & $20,0 \%$ & $40,0 \%$ & $40,0 \%$ \\
\hline Leve & $0,0 \%$ & $75,0 \%$ & $25,0 \%$ \\
\hline Moderada & $25,0 \%$ & $68,8 \%$ & $6,3 \%$ \\
\hline grave & $81,8 \%$ & $18,2 \%$ & $0,0 \%$ \\
\hline
\end{tabular}

Como puede observarse, los datos poseen claras diferencias según se tenga en cuenta la opinión de los terapeutas o la opinión de los propios pacientes. Tomando en cuenta la opinión del terapeuta, siendo apreciable el coeficiente de contingencia encontrado $(0,56, p=0,01)$, los resultados son dispares según la categoría clínica analizada. Así, se podría decir que los grupos de 'no depresión' y 'depresión leve' se ajustan a lo esperable: el $100 \%$ que puntúa como no depresivos son valorados con un progreso terapéutico apreciable. En el nivel de depresión leve, 2/3 partes también son valorados con progreso 
apreciable y $1 / 3$ con progreso intermedio. Sin embargo, en las otras dos categorías algunos datos pueden resultar coritradictorios. Asi, un $56 \%$ de los que puntúan con depresión moderada son considerados por los terapeutas con un progreso apreciable. En el caso de depresión grave casi un $7 \%$ es considerado también con ese tipo de progreso y casi un $50 \%$ se considera que tienen una cierta progresión terapéutica. Menos de la mitad de los que puntúan en el BDI-Il como graves son considerados 'sin mejora' terapéutica.

Cuando se toma en cuenta la opinión del paciente los datos parecen ajustarse más. El coeficiente de contingencia fue de 0,58 $(p=0,01)$. En la categoría de depresión grave ninguno se valora a si mismo como 'bien adaptado', situándose la gran mayoría en la categoria de 'no mejora'. En el caso de niveles de depresión moderada y leve, la categoria más usual es la de adaptación intermedia. Contraniamente a lo que pasaba con la opinión del terapeuta, la categoría más lábil es la de 'sin depresión', donde un $20 \%$ se consideran 'no mejora' producto de su trastomo, a pesar de obtener una puntuación baja en el BDI-II.

Con respecto a los datos para el CBD, en la tabla 6 se resumen los datos de las tablas de contingencia tanto para las opiniones del terapeuta como para los pacientes.

Tabla 6. Tablas de contingencia entre los cuatro grupos clínicos de acuerdo con la puntuaclón obtenida en el CBD y las opiniones de progreso terapéutico emitidos por los terapeutas y ios propios pacientes

\begin{tabular}{|l|r|r|r|}
\hline \multirow{2}{*}{ Grupos clínicos } & \multicolumn{3}{|c|}{ Progreso según los terapeutas } \\
\cline { 2 - 4 } & No mejora & Mejora ligera & \multicolumn{1}{c|}{ apreciable } \\
\hline Sin depresión & $10,0 \%$ & $10,0 \%$ & $80,0 \%$ \\
\hline Leve & $28,6 \%$ & $14,3 \%$ & $57,1 \%$ \\
\hline Moderada & $26,1 \%$ & $65,2 \%$ & $8,7 \%$ \\
\hline Grave & $51,5 \%$ & $42,4 \%$ & $6,1 \%$ \\
\hline
\end{tabular}

\begin{tabular}{|l|r|r|r|}
\hline \multirow{2}{*}{ Grupos clínicos } & \multicolumn{3}{|c|}{ Nivel de adaptación según los pacientes } \\
\cline { 2 - 4 } & No mejora & Nivel intermedio & \multicolumn{1}{c|}{ Mejora } \\
\hline Sin depresión & $2,2 \%$ & $33,3 \%$ & $64,5 \%$ \\
\hline Leve & $7,1 \%$ & $57,1 \%$ & $35,7 \%$ \\
\hline Moderada & $68,0 \%$ & $32,0 \%$ & $0,0 \%$ \\
\hline Grave & $89,2 \%$ & $10,8 \%$ & $0,0 \%$ \\
\hline
\end{tabular}


Los datos con respecto a la opinión de los terapeutas muestran algunas contradicciones ya detectadas con el BDI-II. Así un $10 \%$ de los que obtienen puntuaciones 'sin depresión' en el CBD son considerados sin mejoría por los terapeutas. Un $42 \%$ que según el CBD estaría en puntuación de depresión grave, son considerados por los terapeutas con mejorías intermedias y más de un $6 \%$ como mejorías apreciables.

Cuando se toman en cuenta las opiniones de los propios pacientes, el acuerdo entre juicio clínico y puntuaciones en el cuestionario CBD parece más ajustado. Ahora, ningún caso que de los que puntúan con depresión moderada o grave se consideran a si mismo con una buena adaptación. Al mismo tiempo se mantiene una cierta lógica, existiendo una progresión según el estado de depresión y la consideración de bien o mal adaptados: cuanto más grave es la depresión, mayor porcentaje de casos en la categoría de 'no mejora', mientras que cuanto más leve es la depresión más casos se sitúan en las categorías de 'mejora intermedia' o 'apreciable'.

\section{DISCuSIón}

Hasta aquí hemos presentado los datos sobre la validez de tratamiento del cuestionario básico de depresión. Los datos iniciales muestran que los niveles de depresión evaluados por este cuestionario varian de acuerdo a cómo varia el progreso terapéutico: a menor puntuación en depresión, mayor puntuación en el progreso terapéutico, ya fuera evaluado por los terapeutas como por los propios pacientes. Sin embargo, algunos datos necesitarían alguna aclaración: la comparabilidad entre el BDI-II y nuestro cuestionario, la sensibilidad de ambos inventarios para identificar los distintos estadíos de cambio y su relación con criterios clínicos de mejora.

Los datos proporcionados por el CBD son similares a los del BDI-II, con respecto a la validez de tratamiento. En este sentido, desde el punto de vista de la validez incremental, no parece que este nuevo cuestionario suponga avance alguno con respecto a otros cuestionarios existentes. Es más, en algunos casos parece que el BDI-Il es más sensible a los cambios que el CBD. Se puede observar tanto en unos coeficientes de correlación más elevados (en relación 
con el progreso terapéutico), como en su sensibilidad para monitorizar los tres estadíos de cambio creados (no mejora, mejora ligera o intermedia y mejora apreciable).

La justificación de esas ligeras diferencias a favor del BDI-II puede estribar en una cuestión probabilistica: dado que el BDI-II en sus 21 items evalúa prácticamente 21 áreas diferentes que pueden estar afectadas en los estados depresivos, y el CBD sólo evalúa los componentes de tres áreas (afecto triste, anhedonia y baja autoestima), por lo que es más probable que se observen más cambios cuántas más áreas se evalúen. Si a ello se añade que el tipo de tratamiento que mayoritariamente reciben las personas con depresión de nuestro estudio son psicofármacos, y que el BDI-Il evalúa varios elementos neurovegetativos (fatiga, apetito, sueño...) muy vinculados a la eficacia de esos psicofármacos, la probabilidad de detectar progreso terapéutico con el BDI-II aumenta apreciablemente.

Sin embargo, cuando se toma en consideración criterios clínicos, el análisis anterior puede no estar tan claro: cuando se examinan las puntuaciones medias de los tres grupos de mejora, las puntuaciones medias en el BDI-II indican en todos los casos la presencia de sintomatología depresiva. En el caso de las personas valoradas con de mejora apreciable, las puntuaciones medias son de 21,6 para el criterio de los terapeutas y de 20,6 para el criterio de los propios pacientes, lo que indicaría la presencia de niveles de depresión fronterizos entre depresión leve y depresión moderada. Sin embargo, estos mismos datos para el CBD son de 23,6 y 20,89 respectivamente, lo que indicaría unos niveles de depresión entre ausencia de depresión y depresión leve.

Esta última consideración se observa con mejor claridad cuando se contrastan los niveles de mejora clínicos con las categorías de depresión de los pacientes. Tanto el BDI-II como el CBD muestran una cierta disparidad entre las consideraciones clínicas de los terapeutas y los propios pacientes y las puntuaciones obtenidas en los cuestionarios. Sin embargo, a grandes rasgos, se observan unas peculiaridades diferenciales: mientras que el BDI-II coincide en mayor medida con el criterio de los terapeutas, el CBD coincide mejor con el criterio de los propios pacientes. Las explicaclones para esta situación pueden tener una doble dirección. Por un lado, los clínicos pueden ser muy sensibles a las expresiones de cambios en relación con una serie de áreas 
evaluadas por el BDI-Il y no el CBD: las ya mencionadas quejas de carácter neurovegetativo o las referidas a la distorsión de la realidad (pesimismo, desesperanza...); y pueden estar construyendo su criterio de mejora clínica de acuerdo a la disminución de ese tipo de sintomatología. Por otro lado, los contenidos evaluados por el CBD se restringen a tres áreas que ejemplifican el 'sufrimiento depresivo', en especial el afecto triste y la baja autoestima, lo que podría hacerle más sensible a la percepción de progreso terapéutico por parte de los propios pacientes, en la medida en que consideran que su 'sufrimiento' disminuye.

Estos últimos resultados podrían modificar la idea inicial sobre la escasa o nula validez incremental aportada por el CBD en comparación con el BDI-Il: si bien es posible que desde un punto de vista actuarial el CBD no mejore los datos proporcionados por el BDI-Il; desde un punto de vista clínico el CBD permite monitorizar con mayor precisión los cambios terapéuticos, especialmente cuando se toma en cuenta la opinión de los propios pacientes sobre su estado psicológico.

Con todo, estos datos tienen la provisionalidad que se deriva de al menos tres fuentes importantes, que podrian ser abordadas en futuros trabajos. (i) Los criterios de mejora estadísticos y clínicos tomados por nosotros están en función de la opinión de los terapeutas y los propios pacientes y no dejan de ser opiniones. La disponibilidad de criterios objetivados (inserción laboral, observación sistemática, informes de terceros...) podrlan ajustar mejor los criterios de mejora. (ii) Aquí se han presentado datos de un estudio transversal: se evaluó a un grupo de personas con depresión en distintos estadíos de cambio y se agruparon en función del estadío en el que estaba cada uno. Sin embargo, el proceso depresivo y su sintomatologla pueden ser muy dispares dentro de un mismo grupo, por lo que se hace necesario un seguimiento longitudinal de un mismo grupo de pacientes hasta que remitan clínicamente sus síntomas. $Y$ (iii) por último, se hace necesario contrastar la validez de tratamiento con tratamientos psicológicos y no sólo con psicofármacos. En el caso del CBD, además, es especialmente importante, porque el trabajo psicoterapéutico se suele dirigir a las áreas del 'sufrimiento depresivo' y, por tanto, este cuestionario podría ser un buen instrumento para detectar los cambios.

En resumen, los datos aportados aquí nos indican que el CBD puede tener una buena validez de tratamiento, siendo un instrumento útil para 
monitorizar los cambios que se vayan produciendo producto del progreso terapéutico. Este dato, además, parece especialmente relevante cuando el criterio de mejora terapéutica es la propia opinión de los pacientes.

\section{REFERENCIAS BIBLIOGRÁFICAS}

Asociación Psiquiátrica Americana (2002). Manual Diagnóstico y Estadístico do los Trastomos Mentales. DSM IV-TR. Barcelona, Masson. Asociación Psiquiátrica Americana.(1995). Manual Diagnóstico y

Estadistico de los Trastomos Mentales. DSMIV. Barcelona, Masson. Beck, A., Rush, A., Shaw, B., y Emery, G. (1979). Cognitive therapy of depression. Nueva York, Guilford Press. (trad. Bilbao, D.D.B., 1983). Beck, A.., Steer, R. y Brown, G (1996). Manual for the Beck Depression Inventory-II. San Antonio, Texas, Psychological Corporation.

Beck, A.., Steer, R., y Garbin, M. (1988). Psychometric properties of the Beck Depression Inventory: Twenty-five year of evaluation. Clinical Psychology Review, 8, 77-100.

Beck. A., Ward, C., Mendelson, M., Mock, J. y Erbaugh, J. (1961). An inventory for measuring depression. Archives of General Psychiatry, 4, 561-571.

Borda, M. y Echeburúa, E. (1991). La autoexposición como tratamiento psicológico en un caso de Agorafobia. Análisis y Modificación de Conducta, 17, 993-1012.

Butcher, J., Dahlstrom, W., Graham, J., Tellegen, A. y Kaemmer, B. (1989). Minnesota Multiphasic Personality Inventory-2 (MMPI-2): Manual for administration and scoring. Minneapolis, University of Minnesota Press.

Butcher, J., Graham, J., Ben-Porath, Y., Tellegen, A., Dahlstrom, W. y Kaemmer, B. (2001). MMPI-2 (Minnesota Multiphasic Personality Inventory-2): Manual for administration, scoring and interpretation, revised. Minneapolis, University of Minnesota Press.

Cronbach, L. y Gleser, G (1957). Psychological tests and personnel decisions. Urbana, University of Illinois Press.

González, M., Herrero, M., Viña, C., Ibáñez, l., y Peñate, W. (2004). El modelo tripartito: relaciones conceptuales y empíricas entre ansiedad, depresión y afecto triste. Revista Latinoamericana de Psicología, 36, 289-304. 
Greene, R. (1987). Ethnicity and MMPI performance: A review. Psychology, 55, 497-512.

Gross, K., Keyes, M. y Greene, R. (2000). Assessing depression with the MMPI and MMPI-2. Joumal of Personality Assessment, 75, 464-477. Hathaway, S. y McKinley, J. (1943). MMPI manual. Nueva York, The Psychological Corporation.

Haynes, S. y Lench, H. (2003). Incremental validity of new clinical assessment measures. Psychological Assessment, 15, 456-466.

Hunsley, J. y Meyer, G. (2003). The incremental validity of psychological testing and assessment: Conceptual, methodological, and statistical issues Psychological Assessment, 15, 446-455.

Levitt, E. y Lubin, B. (1975). Depression: concepts, controversies and some new facts. Nueva York, Springer.

OMS. (1992). CIE-10, décima revisión de la clasificación intemacional de enfermedades. Trastornos mentales y del comportamiento. Madrid, TEA ediciones.

Pelechano, V. (1996). Una introducción al modelo de parámetros en personalidad. En V. Pelechano (Ed.), Psicología de la Personalidad. 1. Teorias (pp. 337-368). Barcelona, Ariel.

Pelechano, V. (2000). Teoría sistemática de la personalidad. BarceIona, Ariel.

Peñate, W. (2001a). Presentación de un cuestionario básico para evaluar los síntomas genuinos de la depresión. Introducción. Análisis y Modificación de Conducta, 27, 679-731.

Peñate, W. (2001b). Primer estudio: El cuestionario básico de depresión (CBD): Primeros datos de validación. Análisis y Modificación de Conducta, 27, 733-761.

Peñate, W. (2001c). Cuarto estudio: Revisión del cuestionario básico de depresión: Afecto triste, anhedonia y baja autoestima. Análisis y Modificación de Conducta, 27, 799-809.

Peñate, W. y Almeida, A. (2001). Quinto estudio: Validez discriminante del CBD-rev entre distintos tipos de trastomos depresivos. Análisis y Modificación de Conducta, 27, 811-829.

Peñate, W., Ibáñez, I. y González, M. (2001). Sexto estudio: La estructura final del CBD-rev. Implicaciones para le diagnóstico diferencial con los trastornos de ansiedad. Análisis y Modificación de Conducta, 27, 831-849. 
Peñate, W., Perestelo, L. Y Bethencourt, JM. (2004). La predicción diferencial del nivel de depresión por las variables nivel de actividad, actitudes disfuncionales y estilo atributivo, en función de la puntuación y la medida de depresión utilizada. International Joumal of Clinical and Health Psychology, 4, 27-53.

Peñate. W. y González, M. (2001). Tercer estudio: Validación cruzada de la estructura factorial del CBD. Datos sobre sus características psicométricas. Análisis y Modificación de Conducta, 27, 777-797. Sanz, J., Navarro, M.E. y Vázquez, C. (2003). Adaptación española del Inventario para la Depresión de Beck-II (BDI-II): 1. Propiedades psicométricas en estudiantes universitarios. Análisis y Modificación de Conducta, 29, 239-288.

Sanz, J., Perdigón, L. A. y Vázquez, C. (2003). Adaptación española del Inventario para la Depresión de Beck-II (BDI-II): 2. Propiedades psicométricas en población general. Clínica y Salud, 14, 249-280. Sechrest, L. (1963). Incremental validity: A recommendation. Educational and Psychological Measurement, 23, 153-158.

Spielberger, C., Carretero-Dios, H., de los Santos, M. y Buela-Casal, G. (2002a). Spanish experimental version of the State-Trait Depression Questionnaire (ST-DEP): Trait subscale (T-DEP). International Joumal of Clinical and Health Psychology, 2, 51-69. Spielberger, C., Carretero-Dios, H., de los Santos, M. y Buela-Casal, G. (2002b). Spanish experimental version of the State-Trait Depression Questionnaire (ST-DEP): State subscale (S-DEP). International Journal of Clinical and Health Psychology, 2, 71-89. Steer, R., Brown, G. Beck, A. y Sanderson, W. (2001). Mean Beck Depression Inventory-II scores by severity of major depressive episode. Psychological Reports, 88, 1075-1076.

Steer, R., Clark, D., Beck, A. y Ranieri, W. (1999). Common and specific dimensions of self-reported anxiety and depression: the BDI-Il versus the BDI-IA. Behaviour Research \& Therapy, 37, 183-90.

Storch, E, Roberti, J. y Roth, D. (2004). Factor structure, concurrent validity, and internal consistency of the Beck Depression InventorySecond Edition in a sample of college students. Depression \& Anxiety, 19, 187-89. 


\section{ANEXO \\ PROTOCOLO DE CONTROL (TERAPEUTAS)}

CODIGO DE IDENTIFICACIÓN:

EDAD: GÉNERO:

OCUPACION:

SITUACIÓN LABORAL:

DIAGNOSTICO:

\section{PARA LA PRIMERA OCASIÓN QUE SE CUMPLIMENTE}

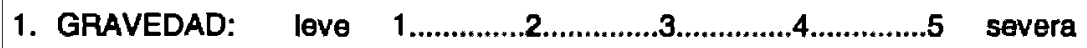

2. PREVALENCIA DEL TRASTORNO:

Menos de un año

Un año o más

Dos años o más

Tres años o más

3. COMORBILIDAD (indicar el trastomo comórbido):

4. ANTECEDENTES FAMILIARES: NO ( )

SI ( ): $1^{9}$ grado ( ); $2^{9}$ grado ( )

5. TRATAMIENTOS RECIBIDOS O QUE RECIBEN:

Psicoterapia

Psicofámacos

Ambos

Otros, ¿cuál?.

6. HOSPITALIZACION POR EL TRASTORNO:

No

SI, sólo en un ocasión

SI, en más de una ocasión 


\section{SITUACIÓN ACTUAL (TERAPEUTA)}

\begin{tabular}{|ll|}
\hline CÓDIGO DE IDENTIFICACIÓN: & FASE: I ( ) II ( ) III ( ) IV ( ) \\
\hline EDAD: $\quad$ GÉNERO: & FECHA (D/M/A): \\
\hline
\end{tabular}

1.

\begin{tabular}{|l|c|c|l|}
\hline Primera consulta & $\mathrm{Si}$ & No & \\
\hline Consultas sucesivas & $\mathrm{Si}$ & No & $\mathrm{N}^{2}$ consulta: \\
\hline
\end{tabular}

2. TRATAMIENTOS:

Psicoterapia: $\begin{array}{ll}\text { NO } & (\text { ) } \\ \text { SI } & (\text { ) }\end{array}$

- Psicoanálisis, terapias dinámicas

- Psicoterapias breves

- Terapia conductual

- Terapias cognitivo-conductuales

- Terapia de reestructuración cognitiva

- Psicoterapia interpersonal

- Terapia racional-emotiva

- Otras, ¿aval?

- Terapia sistémica

- Otras, ¿cuál?

Psicofármacos: NO( )

sí ( )

- Tricíclicos

- ISRS

- ISNA

- ADT

- I-MAO

- Otros, ¿cuál?

3. FASE TERAPÉUTICA.

- Fase de evaluación

- Fase inicial

- Fase de tratamiento

- Fase de seguimiento 
4. SITUACIÓN TERAPÉUTICAACTUAL (marcar la opción).

\begin{tabular}{|l|c|c|c|c|}
\hline $\begin{array}{l}\text { Sin cambios } \\
\text { apreciables }\end{array}$ & $\begin{array}{c}\text { Agün cambio, sin } \\
\text { significación dinica }\end{array}$ & $\begin{array}{c}\text { Agún cambio, } \\
\text { dinicamente significativo }\end{array}$ & $\begin{array}{c}\text { Cambios cinicamente } \\
\text { significativos }\end{array}$ & $\begin{array}{c}\text { En remision total } \\
\text { de sintomas }\end{array}$ \\
\hline & & & & \\
\hline
\end{tabular}

5. NIVEL DE ADAPTACIÓN SEGÚN LA INFORMACIÓN DISPONIBLE (paciente, familiares, otros):

\begin{tabular}{|l|c|c|c|c|}
\cline { 2 - 5 } \multicolumn{1}{c|}{} & $\begin{array}{c}\mathrm{Ha} \\
\text { empeorado }\end{array}$ & $\begin{array}{c}\text { Sin } \\
\text { cambios }\end{array}$ & $\begin{array}{c}\text { Comparable a } \\
\text { antes de la crisis }\end{array}$ & $\begin{array}{c}\mathrm{Ha} \\
\text { mejorado }\end{array}$ \\
\hline 5.1 Adaptación laboral/escolar & & & & \\
\hline 5.2 Adaptación social & & & & \\
\hline 5.3 Adaptación familiar & & & & \\
\hline 5.4 Ocio y tiempo libre & & & & \\
\hline
\end{tabular}

